\section{Chinks of light}

\section{Rodney Loudon}

Atoms and Light: Interactions. By John N. Dodd. Physics of Atoms and Molecules Series. Edited by P. G. Burke and H. Kleinpoppen. Plenum: 1991. Pp.247. $\$ 78$.

THE relationship between the classical and quantum theories of light intrigues almost every physicist trying to reconcile its classical-wave and quantum-particle behaviours. Strong similarities exist between the two theories: Maxwell's equations are valid in both the quantum and classical schemes, and the boundary conditions controlling the behaviours of light beams are common to both. Optical systems are therefore described by identical sets of spatial modes in the two theories, which thus predict the same distributions of intensity in optical experiments. Differences arise only when experiments are performed in which the operator characters of the field vectors in the quantum theory are brought into play by the measurement process. The noncommuting properties of the field operators can then produce such typical quantum-mechanical effects as Heisenberg's uncertainty limitations on the accuracies of measurements and unavoidable perturbations of the measured system.

It turns out that these quantum effects are difficult to observe in practice, and the results of most optical experiments can be perfectly well accounted for by the classical theory of light. Even experimental results that were once thought to demand a quantum interpretation have yielded to theories in which the optical field is treated classically but the full quantum theory is applied to any atoms whose transitions are used in making the measurements, the so-called semiclassical theory. Such is the case with the photoelectric effect, which was regarded as the exemplar of the quantum interpretation, but can in fact be accounted for semiclassically.

Nevertheless some optical effects do require the quantum theory for a quantitative explanation. So although the results of all experiments performed to date are in accordance with the quantum theory of light, only a subset (albeit a large one) accords with the classical interpretation. The nonclassical effects often require feeble light for their observation, where the light has a chance to display its quantum granularity, but such light does not necessarily show quantum effects. For example, careful experiments show that the fringe patterns in a Young or Michelson interferometer do not detectably change however feeble the intensity of light, provided that there is a compensating increase in the recording time. On the other hand, a light beam divided by a simple beam splitter shows extreme particle-like effects, with each incident quantum being directed into either one output arm or the other, but never being separated into two half quanta. Indeed, it is the correlation effects between two or more light beams that provide a rich variety of quantum phenomena.

Dodd is a proponent of the classical theory of light and the semiclassical methods of treating its interaction with atoms. He presents these theories straightforwardly, and I criticize only his insistence on using the ordinary frequency $v$ instead of the angular frequency $\omega$, trivial perhaps, but the resulting high powers of $2 \pi$ complicate many of the equations. The main part of the book explores the extents to which the semiclassical theory can describe results that have been conventionally regarded as needing a quantum interpretation. The

\section{Body building}

\section{Susan E. Evans}

Functional Chordate Anatomy. By Ronald G. Wolff. D. C. Heath and Co., 125 Spring Street, Lexington, Massachusetts 02173, USA: 1991. Pp. 752. $\$ 50$.

How do you breath if you have no diaphragm and your ribs are fused into a rigid shell? Turtles solve the problem by shunting their internal organs around and by changing the volume of soft pockets at the bases of their limbs. Why do cats get stuck in trees? Because their claws are designed to grip on the way up and a cat must learn to come down in reverse.

Functional Chordate Anatomy covers similar ground to its predecessors, beginning with a history of anatomy, and introducing systematics and embryology before launching into detailed accounts of the main body systems. It differs, however, in the breadth of its coverage. The book contains all the classical stories of comparative anatomy - aortic arches, heart and kidney structure, limb patterns and the like - but Wolff has researched his subject well and has included a wealth of additional information previously available only in the primary literature. Each of the main chapters begins with an account of development and ends with a phylogenetic overview. Structure is related to function in the context of individual lifestyles. This is refreshing; some texts leave the impression that non-mammalian amniotes are simply imperfect prototypes of approach is honest, with shortcomings in the semiclassical theory clearly identified. Modifications that are needed to coax the theory towards the desired results and that are sometimes seemingly arbitrary and difficult to justify, are properly discussed. At the end of the book, Dodd describes his novel views about the nature of the classical and the quantum theories.

The book will appeal mainly to those whose interests are in stretching the applicability of semiclassical theory and in finding chinks in the armoury of quantum optical effects that yield them amenable, after all, to semiclassical theory. Most will probably conclude that if they really want to understand the whole range of optical experiments and predict new phenomena to be observed, then they had better come to terms with the quantum theory of light.

Rodney Loudon is in the Department of Physics, University of Essex, Colchester $\mathrm{CO} 4$ 3SQ, UK.

the mammalian end model.

The book is well-organized and clearly written, with good chapter bibliographies and an extensive glossary. It is, to coin a vogue term, 'user-friendly'. A book as comprehensive as this, however, will inevitably contain small errors (wandering labels for example) and omissions. If I have one serious criticism, it is that the text is not always internally consistent. The digits of the bird hand, for example, are numbered 1 , 2 and 3 in one chapter, but 2,3 and 4 in another. There are genuine differences of opinion between palaeontologists, comparative anatomists and developmental biologists on the homologies of these digits, but a note of explanation might have aided the bemused reader. The inconsistency also extends to the systematic section. Wolff presents a long, overly detailed and, in many ways, outdated list of chordate taxa. Here the text is better, being clearly derived from a different source, but in some places it directly contradicts the preceding list, the description of the relationships of frogs and salamanders being an example.

Nonetheless, Wolff is to be congratulated for having produced a very readable book, of use not only to all with an interest in zoology and anatomy, but also to those seeking a wider perspective, such as medical students and developmental biologists. He has shown that comparative functional anatomy is an active and exciting field with plenty of questions left to be answered.

Susan E. Evans is in the Department of Anatomy and Developmental Biology, University College London, Gower Street, London WC1E 6BT, UK. 